\title{
AUMENTO DE RECURSOS DE AGUA: NUEVAS TECNOLOGÍAS Y REPERCUSIÓN SOCIOECONÓMICA EN LA VEGA BAJA Y BAJO VINALOPÓ
}

\author{
Cipriano Juárez Sánchez-Rubio
}

\section{RESUMEN}

La comarca Meridional agraria de Alicante reúne una serie de condiciones objetivamente ventajosas que la convierten en una de las zonas con mayor potencial agrícola del arco mediterráneo español. Todas las condiciones son favorables menos la extensión de la explotación y el déficit hídrico estructural que soporta, agravado por el período de sequía, 1991-1995.

La corrección por economía del agua mediante la nueva tecnología de la desalinización de aguas salobres subterráneas, como alternativa coyuntural para paliar la sequía, permite: mantener en producción la superficie regada, asegurar los niveles de producción y calibre de los productos, ser rentable económicamente, no disminuir la capacidad de trabajo y no provocar pérdidas medio ambientales. No obstante, la solución para erradicar el déficit hídrico, como factor limitante de la actividad económica, pasa por hacer realidad el principio de la intercomunicación de las cuencas hidrográficas, que propone el Plan Hidrológico Nacional.

Palabras claves: Nuevos recursos de agua, desalinización, sequía, pérdidas económicas, conexión de cuencas hidrográficas.

\section{ABSTRACT}

The southern part of Alicante has a series of objectively advantageous conditions which make it one of the areas with the biggest agricultural potential in the Mediterranean arch. All conditions are favourable except for the extension of the exploitation and the water deficit it has to bear, made worse by the drought period between 1991 and 1995 .

The correction by water economy through the new technology of desalting underground briny waters as a temporary alternative to lessen the effects of the drought makes it possible: to keep the irrigated area in production, to maintain production levels, to be profitable from the financial point of view, not to reduce the working capacity and not to cause environmental losses. Nevertheless, the solution to eradicate water shortages as a factor limiting the economic activity must go in the direction of making possible the principle of intercommunication between water basins as is foreseen in the Hydrologic Plan.

Key words: New water resources, Desalting, Drought, Economic Losses, Connection of water Basins. 
En la comarca Meridional agraria de la provincia de Alicante, integrada por la Vega Baja y el Bajo Vinalopó de clima árido y semiárido, el problema de disponer de agua suficiente en cantidad y calidad para no limitar el desarrollo de las actividades productivas es una constante histórica superada por sucesivas alternativas. Como el proceso económico y social se ha fundamentado en la mayor cuantía y variedad de usos de agua, la demanda ha crecido vertiginosamente tanto para atender la actividad agraria, la mayor consumidora, como por el proceso industrial y el desarrollo del sector servicios, especialmente turístico, en expansión.

La necesidad de atender los actuales y futuros requerimientos de agua comporta tanto la mejora en la optimización de la gestión de los recursos (un problema muy difícil y complejo por coincidir elevada demanda y escasos recursos), como la aplicación de nuevas tecnologías capaces de incrementar los caudales (potabilización de aguas salobres y de agua del mar). Y todo ello, sin renunciar a poder compartir los desiguales recursos del territorio peninsular español mediante la intercomunicación de las cuencas hidrográficas.

\section{Aumento de disponibilidad hídrica y cambio en la economía agrícola}

El agua siempre ha jugado un papel importante en la historia agraria de la comarca alicantina, sobre todo, desde principios de siglo a la actualidad. En este territorio confluyen una serie de caracteres físico-ecológicos de estímulos contrapuestos a la expansión del aprovechamiento agrario del suelo. La escasa precipitación, mal distribuida en el tiempo y agravada por la elevada irregularidad, es un factor limitante de la producción agraria que a diferencia de la temperatura se puede corregir. En cambio la fertilidad de los suelos y las favorables condiciones térmicas de la suavidad invernal y las altas temperaturas del verano (factor contrapuesto y positivo) invita a la transformación de secano a regadío. La aplicación del agua a la agricultura garantiza las producciones anuales, aumenta los rendimientos por unidad de superficie y eleva tanto la productividad como el valor de la tierra. El paisaje agrario se modifica enteramente pasando del policultivo de secano (cereal, olivo, almendro etc) a hortalizas, cítricos, cultivos industriales, forrajeras, etc.

La demanda generada para satisfacer la creciente superficie de regadío y demás actividades económicas proviene de los recursos superficiales del río Segura regulados en su cabecera, de las elevación de aguas sobrantes desde 1915, de la explotación de las aguas subterráneas, de los recursos excedentes trasvasados de la cabecera del río Tajo, y recientemente de los caudales proporcionados por la depuración de las aguas residuales.

La agricultura de secano obtenía baja productividad y provocaba movimientos migratorios que aumentaban en los años secos. Por ello, se ha mantenido una lucha constante por el dominio del agua para ampliar y garantizar el espacio transformado por el beneficio económico y social que representa al incrementarse las exportaciones hortofrutícolas, atender la demanda interior y mejorar la balanza comercial. Además, la confluencia en el territorio de otra serie de factores convierten a éste en un recurso con capacidad para ser explotado económicamente. El marco físico se transforma en un espacio socioeconómico evolucionado mediante la realización de infraestructura que lo individualiza, como medio de producción, y le dotan de nueva funcionalidad.

Un hito importante en la ampliación de la superficie regada en la comarca de la Vega Baja fue el Decreto y Orden Ministerial de 25 de abril de 1953, cuyo objetivo básico es ordenar los recursos hidráulicos superficiales existentes en la cuenca y los derivados de la construcción de los nuevos pantanos de Cenajo (1957) y Camarilla (1960). Las nuevas piezas reguladoras suponen un aumento neto de $233 \mathrm{Hm}^{3}$, el $72 \%$ de las disponibilidades anteriores, que mejora el nivel de garantía de los regadíos tradicionales $(21.500 \mathrm{Ha}$. en 
sector alicantino), y la posibilidad de ampliar en $12.500 \mathrm{Ha}$. contiguas a los riegos tradicionales en la forma siguiente: Vega Alta y Media (Murcia) 4.500 Ha. cada una y 3.500 Ha. para Vega Baja. Sin embargo, debido a las favorables condiciones económicas que el regadío reporta, entre seis y diez veces el valor obtenido en la misma extensión de secano, la superficie solicitada afectó a 36.844 Ha., repartidas en la forma siguiente: Vega Alta 12.731 Ha., Vega Media 10.024 Ha. y Vega Baja 14.089 Ha. Es decir, casi tres veces más que la contemplada por la Administración. Una proporción que es cuatro veces superior en las tierras alicantinas.

El desajuste entre la dotación del agua concedida y la superficie solicitada en la Vega Baja motiva la aparición en el nuevo regadío de todas aquellas técnicas que hacían posible economizar agua para lograr abastecer con algún riego al año a todas las tierras (riegos localizados, depósitos reguladores). También la búsqueda incansable de nuevos aportes, mediante la explotación de las aguas subterráneas unida a las extracciones abusivas del cauce del Segura, generando una lucha o guerra por el agua entre los regadíos tradicionales que distribuyen por gravedad las aguas del río (riego de a pie) y los nuevos regadíos que elevan dichos caudales.

La puesta en riego de la superficie solicitada contribuyó a incrementar la productividad agrícola de las tierras beneficiadas, lo que a largo plazo incidiría en una mejor situación económica y social del territorio. Sobre una extensión de 350 Ha., el 10\% de las creadas para riego, de cereales de año y vez con un rendimiento de $2.000 \mathrm{~kg} / \mathrm{Ha}$. en los años abundantes de lluvia, la producción se estimaba en casi dos millones de pesetas corrientes y los gastos en algo más de 1,5 millones de pesetas, con un beneficio de unas 400.000 pesetas que por hectárea equivalía a unas 1.000 pesetas. La transformación de aquella superficie productiva en riego y aprovechada por plantaciones de viñedos en espalderas y parral, limoneros y otros tipos de frutales se obtenía una producción del $1.740 \%$ mientras el beneficio alcanzaba el 4.536\%. El resultado económico de la transformación justifica la inversión en el aprovechamiento del suelo por cultivos de regadío, incluso con aguas elevadas. También por la mejora de orden social materializada en la mayor cuantía de bienes de consumo y paralelo aumento del beneficio empresarial con repercusión en otros bienes y elevación de la renta agraria. Pero sobre todo, por el despegue de los gastos, $1.107 \%$, justificado en la mayor cuantía del número de jornales, incremento de la masa salarial y mayor estabilidad de la mano de obra propensa a la emigración. Proceso de cambio impulsado por la iniciativa pública y privada, en este caso mediante la utilización de las aguas hipogeas.

El gran avance del espacio regado, así como la incorporación de las innovaciones técnicas tendentes a disminuir el consumo de agua y aumentar la producción mediante la práctica de los invernaderos, se realiza en el período de 1960-1980. Los nuevos recursos disponibles provienen de la explotación de los acuíferos. La nueva superficie transformada se asienta en pequeñas explotaciones tecnificadas, altamente capitalizada, de nuevos cultivos y con ventas preferente fuera del país. También grandes empresas cosecheras-exportadoras. La explotación abusiva del agua se tradujo en una alarmante crisis, entendida como escasa rentabilidad de los cultivos en la relación de agua utilizada y su creciente costo y baja calidad (salinización).

La maximización del agua subterránea ha supuesto un desequilibrio territorial porque se ha expandido el regadío para obtener beneficios económicos. Como estos acuíferos no han estado regulados y tampoco han recibido aguas superficiales reguladas, porque están asignadas a superficies concretas ya indicadas y son escasas, se ha producido una degradación con repercusión económica y medio ambiental. No obstante se ha generado una renta agraria de considerable valor, en el período de 1960 a 1980, sin pensar en el futuro. La falta 
de racionalidad se ha traducido en una pérdida de calidad y un mayor déficit hídrico paliado por los envíos regulados excedentes y procedentes de la cabecera del río Tajo. Se culmina de esta forma otra etapa más de aumento de recursos de agua alternativos para no frenar la dinámica productiva agraria de este territorio.

La superficie de riego ha crecido también en la comarca del Bajo Vinalopó mediante la elevación de caudales sobrantes del río Segura desde los primeros años del siglo. Se trata de concesiones de aguas públicas hechas a favor de empresas privadas: El Progreso (1915) y Riegos de Levante (1923, concesionarias de sobrantes en la cola del propio río Segura por un volumen de $5.100 \mathrm{l} / \mathrm{seg}$. y $2.600 \mathrm{l} / \mathrm{seg}$. procedentes de una serie de azarbes, por un total de $7.700 \mathrm{l} / \mathrm{seg}$. Un caudal que nunca se ha llegado a recoger ya que en la práctica el volumen disponible medio se estima sólo en $60 \mathrm{Hm}^{3}$, lo que supone un $24 \%$ de los caudales concedidos debido al elevado grado de regulación de la cuenca del Segura y de la mala calidad del agua recibida. Es la lucha constante por disponer de cualquier tipo y cantidad de recurso agua como el factor económico más eficaz para aumentar el nivel de riqueza y empleo. La potencialidad productiva y económica de esta comarca sería mayor si se dispusiese de caudales suficientes y elevado grado de garantía para desarrollar su agricultura y la posible industria agroalimentaria.

La serie de acciones descritas se refieren siempre al uso agrario del agua tratando promover el desarrollo económico de la zona mediante la ampliación continuada de la superficie regada. La planificación de los aprovechamientos del agua fue propiciada por el Estado al fijar los usos de un determinado caudal de aguas públicas. El de aprovechamiento del río Segura, según el Decreto y Orden de 25 de abril de 1953, es un ejemplo paradigmático de esta planificación donde se evalúan las disponibilidades futuras, las asignaciones a las distintas zonas regables y la superficie objeto de ampliación.

Las acciones mineras del agua en la comarca meridional agraria han provocado un descenso alarmante en los acuíferos que impulsó a acelerar el proyecto de modificar las grandes diferencias de caudales de las vertientes generales. La escasez del recurso en relación con su demanda obliga al Estado a tomar ciertas medidas restrictivas en unas áreas determinadas para ser aplicadas en otras. La planificación implica, por tanto, una adecuada y responsable distribución de las disponibilidades por los poderes públicos en base a los intereses sociales. La necesidad de paliar el déficit hídrico en este espacio y de una forma más amplia en la cuenca del Segura justifica la realización de trasvasar caudales regulados y excedentes de la cuenca del río Tajo.

La política de riegos contenida en los Planes de Desarrollo tenía como objetivo fundamental obtener el mayor crecimiento del producto bruto mediante la aplicación del recurso agua en los territorios más adecuados. Por ello, razones estrictamente económicas, también políticas, propias del modelo desarrollista y de la situación específica del sureste peninsular (escasez de agua en relación con las posibilidades productivas) impulsaron la realización de la obra del trasvase Tajo-Segura. La Ley 52/1980 de 16 de octubre en su disposición adicional primera asigna un volumen de $400 \mathrm{Hm}^{3}$ anuales para uso agrícola. De esta cantidad 127,84 $\mathrm{Hm}^{3}$ corresponden a la comarca Meridional. La aportación supone incrementar en un $55 \%$ el volumen de agua de los regadíos tradicionales y una considerable reducción de las extracciones de los acuíferos sobreexplotados. En la actualidad toda la zona regable está aplicando las aguas transferidas. El aprovechamiento conjunto de estas aguas y las propias han permitido la transformación en regadío de $52.910 \mathrm{Ha}$. en la zona de la Vega Baja y de 24.211 Ha. en el Bajo Vinalopó de las cuales el 34,3\% están atendidas exclusivamente por el agua del trasvase, además de otra gran cantidad de regadío preexistente y mejorado con especial afectación a la superficie dominada por Riegos de Levante Margen Izquierda. 


\section{La agricultura moderna y productiva. Efectos económicos de la sequía}

\subsection{La potencialidad productiva y su dependencia exterior}

El regadío es una potente actividad económica que mediante la producción, comercialización, industrialización y transporte mueve una gran cantidad de mercancías en fresco para atender las demandas del mercado nacional y europeo. La irrigación existente en la Vega Baja y el Bajo Vinalopó es el resultado de un largo proceso de actuación sobre el territorio con especial intensidad en la década de los ochenta, materializada en la ampliación y redotación de una gran extensión de superficie. El suelo se aprovecha, preferentemente, mediante el cultivo de cítricos, hortalizas y frutales frente al secano asentado en los cultivos tradicionales y sometido a una gran irregularidad productiva. Pocas zonas españolas han experimentado un cambio tan radical en su agricultura como el sucedido en este ámbito, conceptualizado en la mejora de las estructuras agrarias de carácter familiar, la presencia de nuevos cultivos, el desarrollo intensivo de las hortalizas, la proliferación de los invernaderos y la sustitución del riego de inundación por el de riego localizado con paralela repercusión en los niveles de renta y empleo.

Las explotaciones agrarias han evolucionado hacia una dinamicidad e intensidad en el uso de la tierra que dista considerablemente de las orientaciones generales aconsejadas por ciertos organismos estatales. Explicar la preferencia por el tipo de aprovechamiento significa comprender su sentido. Los usos del suelo lo son, en gran medida, por el aumento de los rendimientos, el valor total de las producciones, la adecuación a la capacidad del trabajo familiar y la constatación de una cierta garantía de comercializar las producciones. El espacio regado se caracteriza por un dominio de los cítricos, hortalizas y frutales, el $90 \%$, escaso peso relativo de la ganadería y producciones con venta al exterior.

La distribución de los cultivos en las zonas regables es la siguiente: cítricos 53\%, hortícolas $23 \%$, y frutales $14 \%$. Es el dominio de las tierras ocupadas por el limonero. Un cultivo que, al socaire de sus buenas condiciones de adaptabilidad a los suelos calizos y mayor altitud, se ha expandido por los terrenos de baldíos y montes con menos riesgos de heladas de inversión y mayor extensión superficial de la explotación. También, por la poca apetencia de agua, su precio cambiante, la escasa exigencia de mano de obra y su gran facilidad para incorporar los sistemas de riego localizado. Una forma de aprovechamiento de la tierra que sigue siendo socialmente rentable (empleo que genera), económicamente básica en zonas regables concretas (La Pedrera y Riegos de Levante Margen Derecha) y productivamente excedente.

La rentabilidad de las explotaciones de limoneros se encuentra inmersa en un proceso de crisis estructural por el desajuste producido entre los precios de los componentes utilizados en la producción (mano de obra, agua, abonos, insecticidas, etc.) frente a la oscilación de los precios de venta del producto (20 pesetas en 1989; 28 pesetas en 1992; 20 pesetas en 1993 por kilogramo). Un hecho estadísticamente comprobado durante la década de los ochenta que permite un análisis de los niveles de productividad de las explotaciones regadas por los sistemas de inundación y localizado durante el período de 1982-1992. En la primera fecha, sobre un costo de producción de 18 pesetas $/ \mathrm{Kg}$. bajo el sistema de inundación y de 14 pesetas/Kg. en riego localizado con producciones respectivas de $25.500 \mathrm{Kg} / \mathrm{Ha}$ y de $34.000 \mathrm{Kg}$., la rentabilidad neta por unidad de superficie varía de 178.500 a 374.400 pesetas. Cantidades que aparecen modificadas al alza en 1985 cuando los costos para producir la misma unidad de materia se elevan a 21,5 y 16,2 pesetas, respectivamente. En esta fecha la productividad de los tipos de riego es 216.750 y 470.000 pesetas, respectivamente. En 1992 el coste para producir la unidad de peso en riego por inundación se estima 
en 23 pesetas y en riego localizado en 19 pesetas mientras la cotización en el mercado sólo era de 28 pesetas.

La pérdida de capacidad productiva se relaciona con la oscilación del precio percibido por el agricultor. El escaso margen entre el precio de venta y los gastos originados en el sector para obtener el producto arroja un beneficio límite, de manera que el cultivo inicia una reducción de la superficie ocupada. La crisis de la explotación del limonero es consecuencia de la producción excesiva como de la permanencia de los mismos mercados. La superación de esta dificultad se cifra, desde 1990, en la necesidad de reducir considerablemente el volumen de cosecha para evitar el hundimiento de los precios. Esta disminución ya es una realidad con respecto al año anterior en una cuantía del 6\% y mucho más llamativa en 1994 cuando la producción se ha reducido en el 20\% (también interviene la situación de sequía). Un cambio excesivamente rápido y lleno de incertidumbre traducido en la reducción del terrazgo y paralelo descenso de la producción. Además de que la comarca aporta, entre el 85 y 90, es un dato esclarecedor, de la producción total de la Comunidad Valenciana. La saturación de los mercados consumidores, por los envíos de países comunitarios (Italia y Grecia) como por las facilidades otorgadas a terceros países (Marruecos e Israel) y los procedentes del cono sur americano, han repercutido en la capacidad exportadora con incidencia negativa en la renta del agricultor.

Los cultivos hortícolas contribuyen con el 23\%. La alcachofa, afectada por el largo período de sequía (1991-1995), patatas, melón y lechuga son los que más explican en lograr alcanzar aquél porcentaje. El desarrollo espectacular de los invernaderos ha sido el elemento detonante en el despegue de la producción total y su variación espacial con especial afectación al Bajo Vinalopó, confirmado en el valor diferencial del producto bruto por hectárea de superficie cultivada en 1993: Bajo Vinalopó, 725.714 pesetas y Vega Baja, 414.000 pesetas. Cantidades muy significativas tanto con el valor medio de la provincia, entre 1,4 y 2,5 superior, como su propia relación 1,75, aunque el porcentaje del valor de la producción total agrícola sea del 31,2\% en la Vega y del 19,2\% en el Bajo Vinalopó.

Los frutales gozan de larga tradición productiva, sobre todo, el almendro y en menor cuantía el melocotón y la granada. La expansión de aquél ha sido tan grande que se ha establecido como monocultivo en el regadío del Campo de Elche por la escasa demanda de mano de obra y buena adaptación a una agricultura a tiempo parcial motivada por el empleo industrial, turismo y servicios. Una superficie de posible ampliación en base a otros nuevos cultivos, mejora en la comercialización y desarrollo industrial.

Las variaciones de la producción agrícola no son nada más que el reflejo evidente de los cambios que ha experimentado la economía de la zona regable, en los grandes grupos de cultivos mencionados. Las pequeñas explotaciones están cultivadas mayoritariamente por sus propietarios y especializadas en cultivos hortícolas intensivos y de alta rentabilidad. En cambio las explotaciones de mayor extensión y las empresas agrarias diversifican su base productiva mediante el aprovechamiento preferente de cítricos persiguiendo el mayor beneficio neto mediante la reducción del riesgo y el ahorro de mano de obra por el precio de los salarios. Espacio transformado que contribuye poco a un cambio social aunque genera un cierto nivel de empleo. La importancia de la economía agrícola comarcal queda confirmada por los altos valores conseguidos en los diversos conceptos, pero la rentabilidad de las explotaciones pasa por una situación difícil agravada por la falta de garantía de agua y la posible pérdida de mercados. La potencialidad del sector agrícola está cuestionada por el modelo de congelación de precios compensado con subvenciones. Éstas son directas y tendentes a mantener, no elevar, los niveles de ingreso de las explotaciones. El impacto fue negativo sobre los productos más emblemáticos y productivos de la agricultura comarcal: frutas y hortalizas, limitadas en su venta al exterior, el $50 \%$, con $87 \%$ a los 
países de la Unión Europea. Una adversidad que convierte al segmento productivo en dependiente de la coyuntura de la economía internacional.

La cuantía de la exportación depende totalmente de tres conceptos básicos: legumbres y hortalizas (tomates, lechugas, etc.), frutas comestibles (agrios, uva de mesa, melones, etc.) y especias. Durante el período de 1989-1992 estos cultivos representan entre el 91 y 93\% del total de la exportación agrícola. Un porcentaje que aparece limitado por la reminiscencia del período transitorio impuesto por el Mecanismo Complementario de Intercambio al tomate, alcachofa, melón, etc., aunque tales producciones tienen garantizada, prácticamente, su comercialización por la gran tradición de los agentes comerciales y la calidad de los productos.

No obstante, se está produciendo un cambio profundo en las reglas del juego del mercado (liberalización de productos agroalimentarios) donde se impone la competitividad. Un cambio excesivamente rápido y lleno de dificultad que incide en una reducción de la extensión del terrazgo de cultivos hortícolas, en función de la dinámica del mercado (alcachofas, tomate, melón agrios, etc.), y por la dura competencia con otros países oferentes de menor coste en la producción (bajo precio de los salarios y menor coste del agua). Por esto, la agricultura de regadío exportadora está inmersa en una problemática cuyo futuro de las explotaciones técnicamente viables de hortalizas, cítricos y otras producciones deben pasar por una concentración parcelaria, mejora de las estructuras (reducir el costo de la producción), suprimir las trabas administrativas y económicas de la Unión Europea, abaratar el sistema financiero y fomentar la asociación de agricultores con fines comerciales para ordenar tanto la oferta como evitar los precios a la baja. Se trata de encarar el futuro y la incertidumbre que le rodea de una forma inteligente y pragmática para solucionar la problemática de las explotaciones agrarias de la comarca Meridional.

\subsection{Efectos de la sequía sobre los cultivos hortofrutícolas}

La sequía es un fenómeno climático. Ha sucedido en el pasado (años cuarenta, sesenta ochenta), sucede en la actualidad (1991-1995), y, desgraciadamente sucederá en el futuro. Hay que contar con ella y tratar de vencerla con todos los medios posibles. Conceptualizada como un mínimo hidrológico respecto al valor normal, es un desquilibrio pluviométrico en el que es muy difícil predecir el principio y estadísticamente de poca garantía estimar el final. También, en una acepción más científica se la considera como una anomalía negativa del balance hídrico en relación con una situación media. Se confirma, por tanto, que la sequía es una reducción muy significativa de la precipitación media anual, que afecta a los caudales de los ríos y a las reservas de agua del suelo.

No obstante existen sequías que se pueden solucionar en escaso tiempo mediante obras. El déficit de agua se supera incorporando al sistema de abastecimiento y riego, recursos procedentes de suministros alternativos. En este territorio la sequía presenta profundas diferencias en relación a otras zonas del país, de obligada mención: la existencia de un déficit hídrico estructural provocado por el desequilibrio entre las disponibilidades y las demandas (ya puesto de manifiesto), y un déficit permanente, necesitado de solución. Además este espacio soporta coyunturalmente períodos secos (1991-1995), que también afecta a la cabecera del río Tajo como a la del Segura, entre las cuales existe una correlación positiva entre sus entradas anuales, con una varianza explicada del $40 \%$. Esto indica que, en términos generales, tanto los períodos de abundancia como las sequías se van a producir conjunta y simultáneamente en ambas cuencas. Y esto ha condicionado el trasvase de recursos con gran repercusión en la economía agrícola de estas comarcas y de las beneficiadas por el trasvase. 
EFECTOS DE LA SEQUÍA SOBRE LOS CULTIVOS HORTOFRUTÍCOLAS DE LAS COMARCAS VEGA BAJA Y BAJO VINALOPÓ

REDUCCIÓN DE LA SUPERFICIE CULTIVADA EN 1993 CON RELACIÓN A LA MEDIA DEL PERÍODO 1986/92

\begin{tabular}{|lcc|}
\hline CULTIVOS & REDUCCIÓN EN \% & PRODUCCIÓN NO OBTENIDA/Tms \\
\hline Cereales & -27 & 11400 \\
Tubérculos & -20 & 12900 \\
Forrajeros & -2 & 3000 \\
Algodón & -18 & 1000 \\
Limonero & -5 & 10900 \\
Almendro & -7 & 3000 \\
\hline
\end{tabular}

PÉRDIDAS DE COSECHAS EN 1993 POR REDUCCIÓN. RENDIMIENTOS

\begin{tabular}{|lccc|}
\hline CULTIVOS & $\begin{array}{c}\text { Producción } \\
\text { media 86/92 }\end{array}$ & $\begin{array}{c}\text { Producción } \\
\text { en 1993 }\end{array}$ & $\begin{array}{c}\text { Pérdida de cosechas entre } \\
\text { media 86/92 } \mathbf{~ 1 9 9 3}\end{array}$ \\
\hline Hortalizas & 176750 & 157034 & $19716 \mathrm{Tms}$ \\
Naranjo & 216502 & 199810 & $16692 \mathrm{Tms}$ \\
Mandarino & 21532 & 21442 & $90 \mathrm{Tms}$ \\
Limón & 223200 & 198800 & $24400 \mathrm{Tms}$ \\
\hline
\end{tabular}

COSECHA DE 1993 NO COMERCIALIZABLE POR BAJOS CALIBRES

\begin{tabular}{|lccc|}
\hline CULTIVOS & $\begin{array}{c}\text { Producción } \\
\text { de } \mathbf{1 9 9 3}\end{array}$ & $\begin{array}{c}\text { Porcentaje de } \\
\text { bajos calibres }\end{array}$ & $\begin{array}{c}\text { Producción no } \\
\text { comercializable }\end{array}$ \\
\hline Naranjo & 199810 & $20 \%$ & $39962 \mathrm{Tms}$ \\
Mandarino & 21442 & $15 \%$ & $3216 \mathrm{Tms}$ \\
Limón & 198800 & $15 \%$ & $29820 \mathrm{Tms}$ \\
Granado & 21000 & $20 \%$ & $4200 \mathrm{Tms}$ \\
\hline
\end{tabular}

Fuente: Consellería de Agricultura y Pesca.

Al persistir la sequía y no poderse recuperar los embalses se ha llegado a una situación límite en la que los recursos disponibles no son suficientes para atender las demandas. En estas circunstancias la agricultura es la más perjudicada en la lucha por la competencia del agua, al tener una menor capacidad de pago, en función de su rentabilidad. Por tanto, en los casos de sequía el objetivo debe ser la búsqueda de la máxima producción por volumen de agua consumida, entendido como el mayor beneficio económico para el agricultor.

El agua trasvasada del río Tajo a la cuenca del Segura ha servido para crear 74.845 nuevas hectáreas y la redotación de 58.335 Ha., en 1994. La superficie afectada en el territorio de la provincia de Alicante, en el primer concepto, se eleva a 29.628 Ha. 
Cuadro 2

SEGUIMIENTO DE LA SEQUÍA. SEPTIEMBRE DE 1995

\begin{tabular}{|c|c|c|c|c|c|c|}
\hline & \multicolumn{2}{|c|}{ Período Base 1990-1992 } & \multicolumn{2}{|c|}{ Actual } & \multirow{2}{*}{$\begin{array}{c}\text { Valoración } \\
\begin{array}{c}\text { Difer- } \\
\text { Produc }\end{array}\end{array}$} & \multirow{2}{*}{$\begin{array}{c}\text { Pérdidas } \\
\text { Millones }\end{array}$} \\
\hline & Superficie & Producción & Superficie & Producción & & \\
\hline & $\mathrm{Ha}$ & $\operatorname{Tm}(1)$ & $\mathrm{Ha}$ & $\operatorname{Tm}(2)$ & $(1)-(2)$ & de Pesetas \\
\hline VEGA-BAJA & 40635 & 848805 & 33290 & 376235 & 470490 & 17886,73 \\
\hline BAJO VINALOPÓ & 15960 & 196130 & 11080 & 76792 & 119338 & 5819,72 \\
\hline TOTALES & 56595 & 1044935 & 44370 & 453027 & 589828 & 23706,45 \\
\hline
\end{tabular}

Fuente: Consellería de Agricultura. Elaboración Personal.

distribuidas de la forma siguiente: Riegos de Levante Margen Izquierda 20.164 Ha., Saladares de Albatera 1.385 Ha., Riegos de Levante Margen Derecha 1.035 Ha. y La Pedrera 7.044 Ha. La extensión del espacio redotado afecta, principalmente, a Riegos de Levante Margen Izquierda con unas $18.000 \mathrm{Ha}$., beneficiaria de $97,5 \mathrm{Hm}^{3}$ anuales del trasvase. El volumen de agua enviado cada año hidrológico dista muy considerablemente de la cantidad proyectada. En el período hidrológico 1985/1994, solamente en dos años el volumen transferido ha superado los $200 \mathrm{Hm}^{3}$, con un máximo de 206,2 $\mathrm{Hm}^{3}$. Durante los cuatro últimos años hidrológicos, en estrecha relación con la sequía existente, los volúmenes trasvasados para riego han sido: $172,9 \mathrm{Hm}^{3}$ en 1990-1991; $99,7 \mathrm{Hm}^{3}$ en el año siguiente, 56,5 $\mathrm{Hm}^{3}$ al finalizar 1993, y 83,9 durante 1993-1994. Estos volúmenes están estrechamente relacionados con las aportaciones recibidas en el sistema de regulación de la cabecera del río Tajo durante los años hidrológicos 90/91, 91/92 y 92/93 cuyas cantidades fueron $827 \mathrm{Hm}^{3}, 510 \mathrm{Hm}^{3}$, y $459 \mathrm{Hm}^{3}$, respectivamente.

La comarca soporta un déficit hídrico continuado que se está cubriendo con aportaciones de aguas subterráneas y depuradas, en la medida que se puede, cuya falta se traduce en un cúmulo de repercusiones económicas, sociales y ecológicas. La primera confirmada mediante la reducción de la superficie cultivada en 1993 con relación a la media del período 1986/92, con paralela disminución de calibres del producto y descenso de la producción total (cuadro $\mathrm{n}^{\circ} 1$ ). Esta tendencia persiste todavía en septiembre de 1995 con relación al período base de 1990-92 (cuadro $\mathrm{n}^{\circ} 2$ ) en las variables económicas más genuinas del conjunto territorial en la forma siguiente: la superficie regada disminuye en 12.225 Ha. (22\%), la producción en $589.828 \mathrm{Tm}$. (el 57\%) y las pérdidas se elevan 23.706,45 Millones de pesetas. Sin embargo, la afectación interna es desigual ya que en el Bajo Vinalopó la superficie productiva se reduce en un $30 \%$, y en un $18 \%$ en la Vega Baja, lo mismo que la producción, $60 \%$ en aquélla y $55 \%$ en ésta, cuando la relación de la valoración de pérdidas se eleva al 3,07. La segunda, en la pérdida de capacidad de trabajo directa e indirectamente y la tercera por incidencia medio ambiental, daño diferido al arbolado y cultivos sensibles por la sobreexplotación de los recursos subterráneos. Estas mismas características se confirman en el conjunto más amplio de todas las zonas dominadas por las aguas del trasvase durante las campañas agrícola de 1991 a 1994 en la forma siguiente: reducción de la superficie cultivada en el $23 \%$, disminución de la producción en el $42 \%$, pérdidas en pesetas del $75 \%$, descenso de la mano de obra el $18 \%$, endeudamiento creciente del sector y daño medio ambiental difícil de evaluar.

La sequía está provocando el abandono de la tierra por la imposibilidad de disponer de 
agua de riego de forma constante. Los cultivos más representativos de la unidad territorial han reducido su superficie labrada entre el período base y septiembre de 1995 en la forma siguiente: limonero $845 \mathrm{Ha}$., almendro $2.262 \mathrm{Ha}$., tomate $574 \mathrm{Ha}$., alcachofa $887 \mathrm{Ha}$., melón $150 \mathrm{Ha}$., pimiento $386 \mathrm{Ha}$., etc. La inseguridad en la llegada de los envíos procedentes del río Tajo está creando una frustración entre los regantes de la comarca, después de haberse realizado cuantiosas inversiones en relación con las perspectivas creadas por la garantía de los recursos hídricos objeto de trasvase. La sequía, por tanto, afecta a la economía de la explotación agraria ya que los gastos aumentan todos los años (mano de obra, abonos, insecticidas, agua etc.) mientras los ingresos descienden y el beneficio se reduce considerablemente. El enfoque comercial de estas producciones, básicamente hortalizas, depende cada vez más de los compromisos adquiridos para la venta en grandes mercados. La fijación de la producción con antelación y con un precio orientativo según calibre de los frutos convierte a la falta de agua en el factor limitativo, no tanto por la disminución de la cosecha como por la cuota de pérdida de mercado.

El calibre de los frutos comprometido supone utilizar el agua de acuíferos sobreexplotados con alto grado de salinidad que repercute en lo económico y lo ecológico. En el primero de los conceptos por el desigual precio del agua $\left(13,68\right.$ pesetas $/ \mathrm{m}^{3}$ trasvasada y entre 35 y 40 pesetas la del acuífero) y en el segundo por el elevado índice de salinidad. Un mayor costo que unido a la alta conductividad hace prohibitivo su uso en ciertos cultivos como: apio, lechuga, pimiento, etc. por encima de los $3.500 \mathrm{mmho} / \mathrm{cm}$. y los $4.500 \mathrm{mmho} / \mathrm{cm}$. en el melón, siendo altas las pérdidas de calidad si el agua utilizada supera los $2500 \mathrm{mmho} / \mathrm{cm}$. para: alcachofas, habas, patata, coliflor, etc. Esta situación se agrava con la pérdida de tamaño de las producciones, al variar de una media del $70 \%$ de productos de primera categoría y de precio más alto a un $20 \%$, mientras el resto de la producción se distribuye en diferentes niveles con un precio minorizado de hasta cinco veces su valor máximo.

Por esto, el espacio regado se encuentra inserto en una problemática confirmada en una pérdida de interés o progresiva desincentivación de la inversión agrícola (menor competitividad), y aumento del endeudamiento. El suelo cedido al barbecho social raramente se recupera debido al desajuste entre el precio pagado para producir y el precio percibido por los productos. Y es que la ampliación del espacio urbanizado (disminución en $8.110 \mathrm{Ha}$. de las $38.190 \mathrm{Ha}$. de la superficie regable de RLMI), complejos residenciales, expansión industrial y nuevas expectativas de crecimiento urbano (El Triángulo como nueva centralidad) actúan como mecanismos retardatarios no sólo de la disminución del área regada sino como elementos destructores de la superficie ya transformada (autovías, aeropuerto, centros sanitarios y estudiantiles, etc.). Se ha pasado de una situación boyante caracterizada por la alta rentabilidad y estabilidad del mercado a otra donde la tierra regada de limonero está alcanzando un valor de acuerdo con sus posibilidades de producción y precio del producto.

La comarca Meridional acapara durante el período de 1981 a 1991 entre el 48,6 y el 50,4 del valor de la producción total agrícola provincial. Un porcentaje de participación consolidado que en el futuro se incrementará como consecuencia de la reforma de la Política Agraria Comunitaria y los acuerdos GATT, a pesar de sus implicaciones, que afectan a las tradicionales ventajas comparativas del sector hortofrutícola, dominante en el territorio, con gran repercusión socioeconómica. No obstante, la mayor dificultad de supervivencia de la explotación radica en la falta de agua. La sequía que azota el territorio y la falta de agua ha provocado la pérdida de gran parte del arbolado que será necesario plantar con la nueva normalización de agua (de previsión difícil). Además de que un árbol tarda varias campañas para producir. Una reducción del terrazgo que se hace paradigmática en el 
sector de las hortalizas ya que las plantaciones realizadas de cara a la campaña 1995-96 no suponen ni un 5\% de lo normal siendo la zona por excelencia de donde salen los frutos para la exportación y el consumo nacional. Un hecho significativo que es responsable del aumento del precio de los productos, trasladado a los consumidores mediante el Índice de Precios al Consumo, para lo cual se ha llegado incluso a importar productos excedentarios en el campo español.

Sin embargo, la sequía al reducir la oferta ha provocado que la cotizaciones de los cítricos más representativos de la zona se haya triplicado. También, que en la lucha por superar al fenómeno climático adverso se haya impulsado poderosamente un cambio en el sistema tradicional de riego (inundación) por otro (localizado) cuya aplicación supone un ahorro en el gasto de agua y un ahorro en las mermas que se producen durante la distribución. La acción es sinónimo de racionalidad y de modernización del regadío de las viejas huertas tradicionales que tanta riqueza histórica han generado en la provincia. Una necesidad de obligada adaptación de las estructuras hidráulicas a las productivas y comerciales para que la moderna ciencia y tecnología del riego permitan conseguir al agua todo su valor generador de progreso y bienestar. En esta dinámica se encuentra la Vega Baja que cambiará, en una superficie de $6.800 \mathrm{Ha}$. en el término de Orihuela, el riego tradicional por el de goteo.

La reducción de la oferta, escasez de limones, como consecuencia de la larga e intensa sequía ha provocado que las cotizaciones se eleven considerablemente. La campaña 1993-94 ha sido muy buena de precio porque no ha existido producción y la obtenida adolecía de calidad para su venta en los mercados. Sobre la base productiva del rendimiento máximo por unidad de superficie sólo se ha obtenido un $60 \%$ y un $30 \%$ de ello no reúnen la calidad necesaria para una buena comercialización. La industria los ha retirado a 20 pesetas y el resto se empezó pagando a 30 pesetas y ha ido subiendo hasta alcanzar las 80 pesetas en los primeros días de mayo de 1994. Desde entonces las cotizaciones se han estabilizado entre las 50 y las 70 pesetas. El coste de producción se sitúa entre las 18 y las 20 pesetas mientras se considera que el producto es rentable a partir de las 30 pesetas.

La Cooperativa que comercializa para la exportación la mayor parte de la producción del Bajo Vinalopó, indica que los precios para el productor están garantizados en la banda de 55 a 60 pesetas/kilogramo. Incluso se afirma que el limón de segunda, con defectos externos, no comercializado en la campaña anterior, se está consumiendo en mercados de menor poder adquisitivo y se cotiza a unas 50 pesetas. La crisis en que está inmersa el limón, por las dificultades impuestas en la primera fase del período transitorio en el mercado comunitario europeo, debido a la aplicación de precios de referencia como tercer país y tasas compensatorias, puede verse superada. El mercado se está ampliando en base a Japón y los antiguos Países del Este en los que las capas sociales comienzan a disponer ya de un cierto poder adquisitivo. El juego del mercado, no entrar en competencia con el limón de otras zonas alegadas por política de precio, y las favorables condiciones de aquél, a pesar de los precios mínimos que se aplicará al conjunto de los cítricos por el mercado único, permitirá la rentabilidad de las explotaciones en una economía que considera (Decreto 477/1993 de 2 de abril) la dimensión mínima de 5,5 Ha. Todo el proceso descrito pone de manifiesto las favorables condiciones existentes en el territorio para el desarrollo de una agricultura productiva, competitiva, tecnificada y de exportación menos lo reducido de la superficie y la falta de agua.

\section{Necesidad de nuevos recursos de agua: Nuevas tecnologías y beneficio económico}

La escasez de los recursos hídricos, unida al importante incremento de su demanda que viene porduciéndose en los últimos años, ha impulsado la aplicación de las tecnologías de 
potabilización de agua del mar y agua salobre para abastecimiento urbano y en una escala menor para aprovechamiento en riego. La agravante situación de escasez de recursos ha provocado que la práctica de la desalación experimente un desarrollo estimable con tendencia a incrementarse en el futuro. El Ministerio de Obras Públicas, Transporte y Medio Ambiente ha promulgado el Real Decreto 1327/1995, de 28 de julio, sobre las instalaciones de desalinización de agua marina o salobre con el objetivo de regular este tipo de recursos no convencionales, que al incorporarse al ciclo hidrológico se produzca en consonancia con los principios de la Ley de Aguas de 1985.

En el territorio alicantino se ha instalado una serie de plantas desaladoras para aguas de origen subterráneo sitas en varios términos de la Vega Baja $\left(\mathrm{Fig} \mathrm{n}^{\circ} 1\right)$ y en el municipio de Elche (Bajo Vinalopó) para atender la demanda de los cultivos, por falta de aportaciones de agua de riego procedentes del río Segura y los canales del trasvase Tajo-Segura. Esta acción es imprescindible para paliar las pérdidas de la sequía mientras no mejore la situación y se realice el Plan Hidrológico Nacional. Por esto, se hace imprescindible la utilización de las aguas subterráneas existentes en la comarca aunque no son aptas para el riego de cítricos y hortalizas por su elevada conductividad (5,5 a 6 miligramos/litro). Sin embargo sometidas a un proceso de desalinización mediante la técnica de Osmosis Inversa se puede convertir en agua apta para riego.

El elevado precio para producir agua de este tipo durante la década de los ochenta fue un factor que la desaconsejaba como alternativa viable. Las mejoras producidas por los avances tecnológicos con clara repercusión en la disminución del precio por metro cúbico obtenido ha sido un poderoso incentivo para que la Administración mediante el R.D.L. 6/1994 de 27 de mayo declarase de interés general un programa de plantas desaladoras en la cuenca del Segura (Vega Baja). Y es que la desalación de aguas salobres no tiene limitaciones tecnológicas sino económicas. Un coste del agua que es necesario relacionarlo con la rentabilidad de los cultivos más representativos de la unidad espacial que para un tipo de agricultores será la solución permanentemente y para otros una alternativa a la sequía.

El potencial de aguas subterráneas es importante y la concentración de sales totales estimable. Los sistemas acuíferos se extienden aproximadamente sobre los límites de los riegos tradicionales. El carácter de heterogeneidad de los materiales hace difícil evaluar las reservas de agua almacenada durante los tiempos geológicos aunque han sido calculadas en $500 \mathrm{Hm}^{3}$ hasta una profundidad de 100 metros. Los mismos estudios han estimado que los recursos infiltrados por año en el acuífero se estiman entre 75 y $100 \mathrm{Hm}^{3}$. Éstos proceden fundamentalmente de la lluvia, excedentes de riegos, pérdidas en las redes de acequia y por alimentación subterránea lateral, cuya mejor recomendación para uso posterior es la desalinización. La calidad de estas aguas varía considerablemente en una línea que une Callosa del Segura y Benijofar. En el sector meridional de este eje la concentración de sales se sitúa entre el mínimo de 5 gr/litro y un máximo de 15 gr/litro. En el sector septentrional la calidad del agua tiene una salinidad total comprendida entre 1,5 y 4 gr/litro. Existen, por tanto, una condiciones potenciales que debidamente explotadas se convierten en una alternativa valiosa para uso agrícola en este territorio, pero no como medio de solucionar el grave déficit hídrico.

La producción de agua desalinizada para la agricultura mediante el sistema de la osmosis inversa se puede adecuar a las necesidades de cada cultivo en particular con el consiguiente aumento de rendimiento y calidad en los frutos. Las plantas se instalan para mitigar el problema de la sequía en diferentes tipos de explotaciones. La finalidad es ayudar a resolver las dificultades hídricas por las que atraviesa la zona y donarla de aguas de buena calidad. El recurso obtenido es de buena calidad para el riego a coste bajo y asumible por la agricultura intensiva actual entre 25 y 30 pesetas por metro cúbico y un 
Figura 1. Localización de plantas desalinizadoras a la Vega Baja del río Segura.

consumo energético de 1KWA. La tecnología se introduce en la zona como medio para mantener la agricultura existente ante las dificultades de recibir agua de otra cuencas. La contaminación sobre el medio está ausente porque la salmuera irá directamente al mar. Es, por tanto, un tipo de instalación que contribuye a dulcificar el gran problema de escasez y a mejorar la calidad de las aguas.

La justificación del proyecto para disponer de una planta desalinizadora se puede fundamentar en una serie de razones como: obtener escasos rendimientos en los cultivos practicados mediante la utilización de aguas salobres, riesgo eminente de salinización progresiva de los suelos y paralela pérdida de suelo agrícola, conservar la riqueza creada, mantener los puestos de trabajo e incluso aumentarlos en base a que los cultivos son de exportación, ingreso de remesas monetarias a la economía comarcal, regional y nacional mediante divisas y posible potenciación de los cultivos en base a variedades de vanguardia y de primor y ahorro de agua en un 25 ó $30 \%$ por su buena calidad frente a la salina. En definitiva con la solución adoptada se obtiene agua de buena calidad para la explotación de las especies más exigentes en salinidad, consiguiendo mantener la fertilidad de los suelos.

La inversión a realizar es altamente productiva y rentable, tanto desde la perspectiva del ahorro de agua como de la potenciación o incremento de la productividad al garantizar agua de buena calidad para el riego. Ventajas que se acrecientan al garantizar, independientemente de los períodos de escasez o sequía, el suministro necesario en el momento crítico del cultivo. La valoración de la instalación de la planta hay que realizarla en la forma de entender la posibilidad de disponer de agua suficiente o de no tener para cumplir 
Cuadro 3

DESALINIZADORAS

\begin{tabular}{|c|c|c|c|c|c|c|c|}
\hline $\begin{array}{l}\text { Entidad de } \\
\text { Riego }\end{array}$ & $\begin{array}{l}\text { Superficie } \\
\text { (Has.) }\end{array}$ & $\begin{array}{c}\text { Inversión } \\
\text { Solicitada } \\
\text { 000 Ptas. }\end{array}$ & $\begin{array}{c}\text { Caudal } \\
\text { 1/seg. }\end{array}$ & $\begin{array}{l}\text { HM }^{3} / \\
\text { Año }\end{array}$ & $\begin{array}{l}\text { Inversión } \\
\text { Valorada } \\
\text { 000 Ptas }\end{array}$ & $\begin{array}{c}\text { Subvención } \\
000 \text { Ptas. }\end{array}$ & $\begin{array}{c}\text { Eliminación } \\
\text { Salmuera }\end{array}$ \\
\hline $\begin{array}{l}\text { Agrícola del } \\
\text { Mediterráneo }\end{array}$ & 177 & 117092 & 20 & 0,63 & 80000 & 32000 & Drenaje-Mar \\
\hline $\begin{array}{l}\text { C.R. Río } \\
\text { Nacimiento }\end{array}$ & 300 & 115000 & 50 & 1,58 & 130000 & 52000 & Directo-Mar \\
\hline $\begin{array}{l}\text { C.R. Torre } \\
\text { Miguel }\end{array}$ & 1600 & 89200 & 50 & 1,58 & 140000 & 56000 & Directo-Mar \\
\hline $\begin{array}{l}\text { C. R. Campo } \\
\text { Salinas }\end{array}$ & 2000 & 316100 & 100 & 3,15 & 340000 & 136000 & $\begin{array}{l}\text { Emisario- } \\
\text { Jacarilla }\end{array}$ \\
\hline $\begin{array}{l}\text { C. R. Santo } \\
\text { Domingo }\end{array}$ & 1900 & 373900 & 60 & 1,89 & 210000 & 84000 & $\begin{array}{l}\text { Emisario- } \\
\text { Jacarilla }\end{array}$ \\
\hline $\begin{array}{l}\text { C. R. San } \\
\text { Miguel }\end{array}$ & 1530 & 285000 & 100 & 3,15 & 340000 & 136000 & $\begin{array}{l}\text { Emisario- } \\
\text { Jacarilla }\end{array}$ \\
\hline TOTALES & 7507 & 1296292 & 380 & 12 & 1240000 & 496000 & \\
\hline
\end{tabular}

Fuentes: Empresas. Datos de Campo. Elaboración Personal.

las demandas en su lugar y tiempo para cualquier actividad. También, el carácter de autogestión por las entidades propietarias y la garantía de coste.

La instalación de una serie de plantas desalinizadoras en la Vega Baja para atender a una superficie de $7.507 \mathrm{Ha}$. (cuadro $\mathrm{n}^{\mathrm{o}}$ 3), de preferente uso del suelo por cítricos, y de menor caudal para invernaderos particulares (pimiento, tomates, flores) del Campo de Elche (30 m³/día y $60 \mathrm{~m}^{3} /$ día), es motivo de análisis económico entre coste y beneficios marcado en los límites reales de la modernización del regadío (riego localizado). La inversión solicitada se aproxima a los 1.300 millones de pesetas que produce un caudal en litros/segundo de 280 , equivalente a casi $12 \mathrm{Hm}^{3} /$ año, una inversión valorada en $1.240 \mathrm{mi}$ llones de pesetas y subvención sobre ésta del $40 \%$.

El proceso de desalinización consiste en presurizar una solución salina contra una membrana semipermeable, en magnitud suficiente para facilitar el paso del agua para su uso. La utilización comercial y a gran escala de esta técnica para incrementar los recursos hídricos es relativamente reciente. Los particulares, Corporaciones locales o comunidades de usuarios, podrán solicitar al organismo de cuenca correspondiente una autorización para desalar agua salobre o del mar cuando dicho recurso se destine a su propio consumo o a la prestación de un servicio público de su competencia (Artículo 4, apartado 1). En dicha autorización se establece, el plazo de duración de la misma y los usos a los que se podrá destinar, sin que el agua obtenida pueda ser aplicada a otros distintos. En circunstancias especiales debidamente justificadas (Art. 4, apartado 2 y 4), el MOPTMA podrá autorizar actividades de desalación a particulares o a comunidades de regantes que suponga para el titular un volumen total anual de agua mayor de 500.000 metros cúbicos anuales.

En el marco legal de referencia y a efecto de modelo se analiza el balance de actuación de una instalación de planta desaladora para aguas de origen subterráneo, sita en la unidad Meridional agraria. La finca rústica abarca en la actualidad una superficie de $170 \mathrm{Ha}$. 
Cuadro 4

CARACTERÍSTICAS DE LOS POZOS

\begin{tabular}{|lccccc|}
\hline & Pozo $\mathbf{~}^{\mathbf{0}} \mathbf{1}$ & Pozo n $^{\mathbf{0}} \mathbf{2}$ & Pozo n $^{\mathbf{0}} \mathbf{3}$ & Pozo no $^{\mathbf{4}}$ & Pozo no $^{\mathbf{0}}$ \\
\hline Cota & 28 & 24 & 22 & 26 & 22 \\
Profundidad & 40 & 24 & 28 & 42 & 36 \\
Nivel Estático & 17 & 14 & 11 & 22 & 18 \\
Nivel Bombeo & - & 20 & 24 & 35 & 30 \\
Caudal & 6 & 5 & 2 & 6,2 & 7,03 \\
Conductividad & 5,5 & 5,5 & 5,5 & 6 & 5,5 \\
\hline
\end{tabular}

Fuente: Datos de Campo.

plantadas de cítricos y distribuidas en la forma siguiente: limoneros $100 \mathrm{Ha}$.; naranjos $25 \mathrm{Ha}$ y mandarino $45 \mathrm{Ha}$., regadas en su totalidad por el sistema de goteo. La explotación se encuentra en un $75 \%$ en plena producción. El objetivo es desalinizar aguas salobres de origen subterráneo procedente de cinco sondeos de la finca para producir $1.700 \mathrm{~m}^{3} / \mathrm{d} i ́ a$ de agua permeada. La acción pasa por ser la mejor iniciativa para paliar las pérdidas originadas por la sequía.

Las características de los cinco sondeos son las siguientes: la cota oscila entre 22 y 28 metros, la profundidad de extracción varía de 24 a 42 metros, el nivel estático será comprendido entre 11 y 22 metros, el nivel de bombeo entre los 20 y 35 metros, el caudal de 2 a 7 litros/segundo y la conductividad de 5,5 a 6 gr/litro (Cuadro $\mathrm{n}^{\circ} 4$ ).

La planta desalinizadora se diseña para producir $1.700 \mathrm{~m}^{3} /$ día de agua permeada, equivalente, a $620.500 \mathrm{~m}^{3} /$ año que corresponde a 19,68 1/seg. de caudal instantáneo, mientras el caudal de alimentación es de 26,23 1/seg. ya que sólo se aprovecha el $75 \%$ de la cantidad total y el 25\% como salmuera. Este vertido o parte residual (Art. 5) es conducido al mar Mediterráneo a través de una tubería de desagüe que la Confederación Hidrográfica del Segura ha instalado para evacuación de aguas de drenaje. La operación se realiza mediante un tubo de casi 6.000 metros de longitud capaz de conducir $15 \mathrm{l} / \mathrm{seg}$. de salmuera desde la planta al colector de la CHS. La salmuera producida, con una salinidad media de 18,5 gr/l. de sales totales, no afecta negativamente a las aguas del mar. Su incorporación a éste a través de las Salinas de Torrevieja con un total de sales de $37 \mathrm{gr} / \mathrm{l}$. de agua sólo contribuye a disminuir el nivel de concentración. La incidencia del vertido de la salmuera al mar, 6,55 1/seg., no afecta a la fauna marina porque sus aguas aportan una concentración en sales muy inferior a las propias del mar. Una obra que no produce impacto ambiental, pero de obligado estudio (EIA), como consecuencia clara de la importancia que adquieren los procesos de urbanización y desarrollo industrial, a los efectos de evitar deterioros irreversibles y costosos.

La puesta en funcionamiento de este dispositivo tecnológico comporta un análisis de la rentabilidad financiera de dicha inversión. Cuando se habla de desalación hay que hacerlo con los precios en la mano: 25-30 pesetas por metro cúbico de agua salobre sin contar amortizaciones y transporte del volumen al depósito receptor de las aguas brutas de los pozos. Para otros investigadores este precio sobre los mismos conceptos varía de 22 a 35 pesetas por igual cantidad de agua presurizada. Sin embargo, no hay que hacerse trampa con los números: el agua desalada es una alternativa, en muy pocas ocasiones y en cantidades limitadas, para la agricultura. La inclusión de todos los conceptos que participan de manera diferente en el precio final (energía, depósito regulador, amortización, 
reactivo, valor del agua que se pierde como salmuera, etc.) elevan el precio entre 60 y 75 pesetas por metro cúbico. La del trasvase Tajo-Segura es 18,23 ptas. $/ \mathrm{m}^{3}$ para regadío y puesta en el canal, que como máximo por distribución y gastos de funcionamiento puede llegar a ser de 30 a 35 pesetas por metro cúbico.

La relación por cociente entre el valor de la producción y el coste de los medios de producción arroja un valor positivo y significativo. En la superficie de una hectárea con plantación de cinco por cinco metros existen 400 árboles. La necesidad anual de agua para hacer producir un árbol de limonero es de $10 \mathrm{~m}^{3} /$ año que por 60 pesetas supone un gasto de 600 pesetas. La producción media se estima en $100 \mathrm{Kgr}$. que a un precio de 50 pesetas obtiene un monto de 5.000 pesetas. El coste del agua es, por tanto, del orden del $12 \%$ de la producción final agraria. Sobre la base de estos valores simplificados el gasto originado por hectárea para asegurar la producción es de 240.000 pesetas y el valor de la producción final 2.000.000 de pesetas. Ésta de rentabilidad mejora sensiblemente en el naranjo y el mandarino y, sobre todo, en el aprovechamiento del suelo por los cultivos de invernadero debido al precio más elevado percibido por los agricultores.

El beneficio económico se consigue al tomar en consideración que la garantía de aportar agua suficiente de buena calidad (el agua entra con $4 \mathrm{gr} / \mathrm{l}$. de salinidad y sale después del proceso con $0,2 \mathrm{gr} / \mathrm{l}$.) asegura las cosechas y obtiene los rendimientos máximos de productos por unidad de superficie. La evolución de este parámetro (disminución de los rendimientos en las cosechas por escasez de agua o empleo de recursos de mala calidad) lo determinamos por la diferencia entre ingresos y gastos: 268,5 millones de pesetas; gastos totales 99,1 millones de pesetas y beneficio bruto 177,4 millones de pesetas. Los ingresos se descomponen en la forma siguiente: superficie de limonero 150 millones, de naranjos 37,5 millones y mandarinos 81 millones. Estos resultados se configuran sobre la base de el $75 \%$ de la superficie de cada cultivo, la producción media de 40.000 $\mathrm{Kgr} / \mathrm{Ha}$. y un precio de 50 pesetas para limones y naranjas y de 60 pesetas para mandarinas. El balance arroja un beneficio de 177,4 millones de pesetas.

A la vista de tales resultados, que se pueden ver ligeramente modificados en función del juego del mercado por la variación de los precios, la desalación de agua salobre para aplicar a cultivos hortofrutícolas en la comarca Meridional agraria de la provincia de Alicante (Vega Baja del Segura y Bajo Vinalopó) es altamente positivo. Y es que sin agua no se puede cultivar y los resultados serían negativos. Por otro lado, la aplicación de esta tecnología ayuda a conservar la riqueza creada, a mantener e incrementar los puestos de trabajo y a conformar explotaciones rentables. De esta forma, a la vista de lo que antecede se puede deducir que una reducción en las cosechas por falta de agua o empleo de las de mala calidad disminuiría la producción en mayor cantidad que el importe de las obras necesarias de realización. Por razones obvias la inversión realizada tiene una gran repercusión económica y social en el marco del este territorio. No obstante, consideramos que esta forma de paliar la sequía no es la panacea porque tampoco se pueden agotar los acuíferos. Se trata de tener una garantía de recursos para un determinado período de sequía ya que el conjunto de las aguas depuradas y desalinizadas no llegan a cubrir el $25 \%$ de las demanda.

La política a seguir sería la formación de un regadío de tipo mixto que incorporase todas las mejoras tecnológicas (nuevas membranas, menor necesidad de energía, eficacia de riego al $95 \%$ etc.) tendentes a solucionar el problema de la sequía, mediante la práctica de la potabilización, pero en cuanto se disponga de agua pública, utilizar a ésta que es más barata ( 30 pesetas del trasvase frente a las 60 de la desalinización) en una proporción de siete partes del trasvase frente a tres de la potabilización. La combinación de ambas componentes permitiría un mayor nivel de garantía de disponibilidad de agua y mucho más barata con clara incidencia en el nivel de vida del medio rural. 
Sin embargo, en nuestra opinión, la erradicación del déficit existente y el previsible, a pesar de las políticas de ahorro y gestión de la demanda, de optimización del uso conjunto de las aguas superficiales y subterráneas y de la máxima utilización adicional de los recursos no convencionales de las propias cuencas, sólo se solucionará mediante el criterio de una política de aguas asentada en el principio de la intercomunicación de las cuencas hidrográficas previstas en el Plan Hidrológico Nacional.

El problema del agua se podría resolver mediante un mecanismo, ciertamente complejo, que estableciera un precio por unidad. En este momento el agua no tiene precio en España. Lo que se paga es su transporte o canalización. De esta forma se cedería más fácilmente que ahora, a pesar de que el agua es un bien público, y no imposibilita que se le ponga precio. Un tema poco debatido pero necesitado de una participación de los diferentes grupos, ya que uno de los problemas de la ordenación de territorio es el mejor uso posible de éste elemento del espacio en función de las necesidades de la sociedad. Un recurso de cuyo conocimiento se preocupan: ingenieros, juristas economistas, geógrafos, ecologistas, usuarios del agua y políticos. Y es que, el modelo de desarrollo de una gran parte del país no se puede sostener sin transferencias hídricas. Sin éstas, sería necesario una reorientación de dicho modelo, con el consecuente coste económico y político, debido a que las actividades productivas existentes en éstos no pueden desarrollarse si no cuentan con sus ventajas comparativas específicas.

En conclusión: tras el análisis realizado, se puede afirmar que, la unidad territorial de la comarca Meridional agraria reúne una serie de condiciones objetivamente ventajosas que la convierten en una de las zonas con mayor potencial agrícola del marco mediterráneo español. Todas las condiciones son favorables para mantener la función de producción agrícola menos el déficit hídrico que soporta. La corrección por economía del agua, nuevas aportaciones, reutilización, desalación, etc., permitirá recuperar las ventajas comparativas en los mercados europeos.

\section{Bibliografía}

CABEZAS, F.: Impacto de la estructura estocástica en el planeamiento y la gestión: el sistema TajoSegura. (Mecanografiado).

CANALES, G y JUÁREZ, C. (1994): «Nuevos regadíos en el Bajo Segura: El modelo referencial de San Onofre-Torremendo», (1953-1992), Investigaciones Geográficas, núm. 12; pp. 215-237.

JUÁREZ, C. (1991): Planificación hidrológica y desarrollo económico: el trasvase Tajo-Segura, Instituto de Cultura Juan Gil-Albert. Alicante.

JUÁREZ, C. RAMÓN, A. PONCE, G. y CANALES, G. (1993): «Las agriculturas alicantinas», en PEDREÑO, A. Estructura Económica de la Provincia de Alicante. Diputación Provincial, Alicante, pp. 147-171.

MEDINA, A. et all: Las aguas subterráneas de la provincia de Alicante. Diputación de Alicante e Instituto Geológico y Minero de España. (Mecanografiado).

PÉREZ, F y PÉREZ, F.: Informe sobre la repercusión socio-económica de la sequía en las zonas regables del trasvase Tajo-Segura. (Mecanografiado).

PRATS, D. (1993): Desalinización de agua salobre y agua del mar. Una alternativa para obtener agua de calidad. Canalobre, n 27; pp. 89-116. 